\title{
Proposal and Implementation of MPLS Fuzzy Traffic Monitor
}

\author{
Anju Bhandari \\ Computer Science and Engineering \\ Thapar University, Patiala \\ Punjab, India
}

\author{
V.P.Singh \\ Computer Science and Engineering \\ Thapar University, Patiala \\ Punjab, India
}

\begin{abstract}
Multiprotocol Label Switched Networks need highly intelligent controls to manage high volume traffic due to issues of traffic congestion and best path selection. The work demonstrated in this paper shows results from simulations for building optimal fuzzy based algorithm for traffic splitting and congestion avoidance. The design and implementation of Fuzzy based software defined networking is illustrated by introducing the Fuzzy Traffic Monitor in an ingress node. Finally, it displays improvements in the terms of mean delay $(42.0 \%)$ and mean loss rate $(2.4 \%)$ for Video Traffic. Then, the resu1t shows an improvement in the terms of mean delay $(5.4 \%)$ and mean loss rate $(3.4 \%)$ for Data Traffic and an improvement in the terms of mean delay(44.9\%) and mean loss rate $(4.1 \%)$ for Voice Traffic as compared to default MPLS implementation.
\end{abstract}

Keywords-Multiprotocol Label Switched Networks; Fuzzy Traffic Monitor; Network Simulator; Ingress; Traffic Splitting; Fuzzy Logic Control System; Label setup System; Traffic Splitting System

\section{INTRODUCTION}

Multiprotocol Label Switching Network (MPLS) is one of the promising architecture of the future internet. It has proven to be highly reliable. MPLS provides traffic engineering and virtual private network (VPN) services that lead to solving problems relating to traffic congestion. Currently, these network services depend upon protocols like Resource Reservation Protocol (RSVP), Constraint-based Label Distribution Protocol (CR-LDP), Open Shortest Path First (OSPF) and the extensions for the resource reservation protocol (ERSVP) [1-4].

However, on the same note, all routers must enable new protocols to support any new network services. Extensive simulation using NS-3 and contemporary research for optimizing the high speed network. The tests were conducted to reach optimal conditions for large volume traffic network scenarios. Since, these scenarios are known to be computationally and resource intensive, there is a continuous need for innovation achieving faster response time in managing traffic splitting and congestion avoidance.

However, the main stress is on traffic splitting, which is mainly done dynamic as per traffic conditions and available paths. Multiprotocol architecture provides various features such as Quality of Service (QoS), Architectural Scalability, Differentiated Services (DiffServ), Integrated Services
(IntServ), Explicit Routing and high quality End-to-End (E2E) service for Virtual Private Networks (VPNs).

In this research work, it is illustrated with the architecture and study of improving the performance of transmission through ingress node [7,8]. Ensuring 100\% E2E QoS that makes the network to select the most feasible path in terms of load, delay, utilization rate and link capacity.

The framework proposed till now is lacking in high dynamic traffic scenario. So, there is a need to overcome the main limitations discovered by researchers [9-19]. A deep investigation for analysis of high volume traffic and improving existing algorithms on traffic splitting and congestion avoidance is done.

Constraint based routing or multiple path selection can fail due to no decision making component in software defined networks. It gives high node to node QoS for video rendering applications. The need of the path selection process considers the combination of QoS and Traffic Engineering (TE) constraints dynamically. Therefore, this paper presents experience and fuzzy-based rules for traffic management in Software Defined Networks. This architecture ensures high QoS for video applications.

A virtual environment was created to perform simulations for understanding the bottlenecks in traffic management and building a solution based on this analysis. The framework consists of NS-3 and Fuzzylite.

It presents the integration of the Fuzzy Logic Control System with NS-3 to simulate and model assumed network model. The MPLS module was extended to achieve highly optimized traffic routing. The parameters are defined for standard simulations performed to analyze the MPLS network formation. The Fuzzy logic Control System (FLCS) is sited at ingress node. FLCS is composed of two sub fuzzy control systems ie. Label Switched Path setup System (LsS) and Traffic Splitting System (TSS) as shown in the Fig.1.

LsS selects LSPs according to two input fuzzy metrics (delay and load) [16, 17] and gives output fuzzy metric LsS_value. Load and Delay are suitable QoS factors on which selection of appropriate path depends. TSS performs the traffic splitting among the computed paths, according to the two input fuzzy metrics (utilization rate and link capacity) and gives output fuzzy metric TSS_value. 


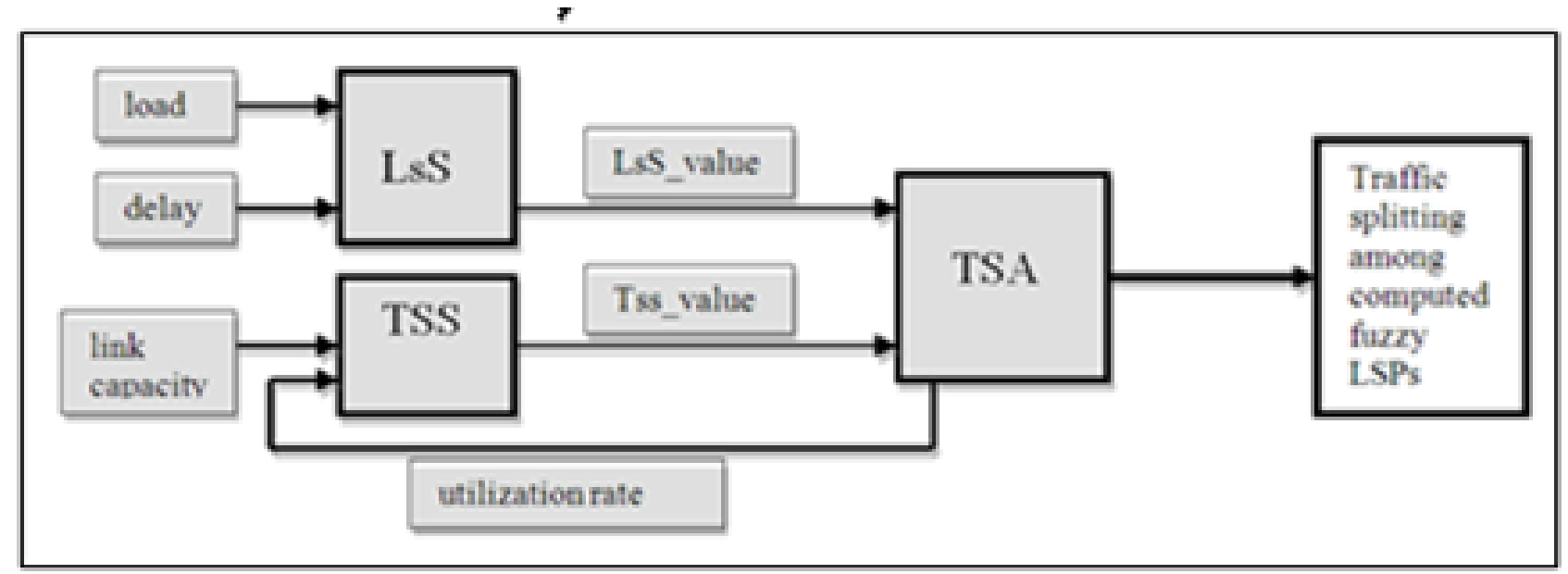

Fig. 1. The Fuzzy Logic Control System consists of LsS and TSS as fuzzy controllers

An appropriate decision of traffic splitting is performed among the computed number of LSPs required by Traffic Splitting Algorithm (TSA). The computed fuzzy based LSPs for forwarding packets are obtained to avoid the situation of underutilization and over utilization of paths. None of the paths remains idle for longer time and proper utilization of resources takes place. Hence, it is better for congestion to be prevented rather than corrected. Implementation of FL using Mamdani Fuzzy Inference System [31] evaluates final decisions as LsS_value and TSS_value. The available number of rules in the Rule Base matrix LsS and TSS represents intermediate situations and provides the control mechanism with a highly dynamic action.

The section II discusses the current scenario of the problems related to traffic splitting and motivation for design of an intelligent system in MPLS networks. We describe the model of our proposed approach in detail in the section III. Section IV, studies the performance evaluation, and finally section V concludes the research paper. We strongly believe that our proposal will considerably helps in solving the congestion problem in MPLS networks. Since, the measurements of the input parameters (ie. Load, delay, link capacity and utilization rate) can be imprecise due to dynamic nature of traffic. In fact, the proposed scheme provides a way forward to a more robust and efficient form of multimedia streams, which is highly needed as the demand grows for better services.

\section{THE CURRENT SCENARIO}

Currently, network QoS attributes are most important for real-time applications. These are not addressed in the path selection process.

As, MPLS is not able to provide intelligent routing, which is a fundamental component of high volume multimedia traffic [4]. The implementation of RSVP with MPLS successfully enables the reservation of bandwidth on a router for each LSP. However, it does nothing to solve the variable demands of multimedia services. With these solutions, MPLS is not able to provide $100 \%$ guarantee on the QoS with respect to high volume incoming traffic. Choosing the best suitable routing algorithm for network is the key factor determining the network lifetime [5]. Moreover, heterogeneity of the nodes in the network also plays a vital role in the network operations [6]. Hence, there is a need to look for a better solution.

\section{A. Technology background}

OSPF is opted for Multiprotocol Label Switching with traffic engineering (MPLS-TE). It is introduced in RFC3630 [7] which work using the OSPF opaque Link State Advertisement (LSA). It builds an extended TE link state database (TEDB), for signaling Extended Resource Reservation Protocol-Traffic Engineering (RSVP-TE). It supports intra domain, hard state connection oriented - set up / take down, provisioning, recovery and many other features. The LSA spread the attributes of TE link among the network. RFC4090[8] performed work regarding routing protocols and redesign to give MPLS Fast-Reroute (MPLS-FRR) which is still not a reliable solution for such a large scale data center networks.

From the mentions of the literature survey [9-15], the analysis of related work shows that although the applicability of this fuzzy approach needs to be put to extensive tests. We can further conclude that it has gained much attention recently that till date OSPF has not been replaced with any better algorithm which can produce better results.

\section{MOdEL Formulations}

After conducting systematic literature survey and studying associated matter related to the problem area. In this work, we propose a fuzzy based solution that does not change the existing conventional algorithms but uses operations of them in order to provide a system that will improve performance. The selection of the fuzzy logic method was based on the simplicity and the fact that since it processes experts defined rules governing the routing system. It can be modified \& tweaked easily to improve the performance. Analysis of the state of the art in it reveals that there is no protocol designed and evaluated. Hence, in this paper, we use the abilities of FL to propose a technique for designing a fuzzy based traffic conditions. In a dynamic network environment, it is difficult to build choice in choosing a path where traffic congestion will be least possible. There are many fuzzy factors responsible to get the best one for a flow. The goal and the 
constraints are fuzzy in nature. Path selection and traffic splitting among selected paths is a decision making problem in a fuzzy network environment. To deal quantitatively with an impression, we employed fuzzy theory to define fuzzy goals or fuzzy constraints precise as fuzzy sets and get fuzzy decisions. Therefore, Fuzzy Logic Control System (FLCS) is proposed to select paths and then traffic splitting among them. Since, there are many available paths for the same source node and destination nodes. So, this approach first gives the list of paths in preference order. Secondly, the traffic will be transmitted among the number of paths required, which is obtained from the controller itself.

\section{ARCHITECTURE}

\section{A. Background and Concepts}

Fuzzy Traffic Manager (FTM) is a novel an intelligent controller resides in an ingress node of MPLS domain as shown in Fig. 2. It works in the LSP selection component. The Label Distribution Protocol (LDP) takes the output of the proposed controller and transmits it to routing protocol (RSVP-TE, OSPF).

The architecture has the following features:-

1) High speed network

Domain consists of two kinds of nodes edge nodes and core nodes. An edge node, is called as label edge router. The ingress label edge router sends packets to the domain and egress label edge router receives packets from the domain. The intermediate nodes are core nodes which perform packet forwarding.

\section{2) Fuzzy Logic Control System}

The proposed fuzzy traffic manager resides in the ingress node works on the execution of Fuzzy Logic Control System (FLCS). The Fig. 1 is holistic view of work done, as seen FLCS consists of two fuzzy controllers LsS and TSS. LsS develops intelligent rules using QoS constraints of traffic flow. It gives the SLSPs as the matrix of LSPs in preference order to be used for routing. TSS develops intelligent rules using TE constraints of traffic flow. It computes the NLSPs as the matrix of number of required LSPs. [16] defines completely mathematically how fuzzy logic will be applied to proposed controllers.

LsS rule base is a set of inputs (delay (D), load(L)) with their five linguistic values (Very Low (VL), Low (L), Medium (M), High (H), Very High (VH)) and output LsS_value with their seven linguistic values (Zero (Z), Tiny (T), Very Small (VS), Small (S), Big (B), Very Big (VB), High (H)) forms at most $5 * 5=25$ possible combinations. Hence, optimized decisions are made using developed 25 rules (R1, R2, and R3...R25) as shown in Fig. 3, which are maximum possible cases for the selection of LSPs under different situations.

This FMM (LsS-Fuzzy Rule Base Matrix) provides large number of options to obtain best routing decisions.

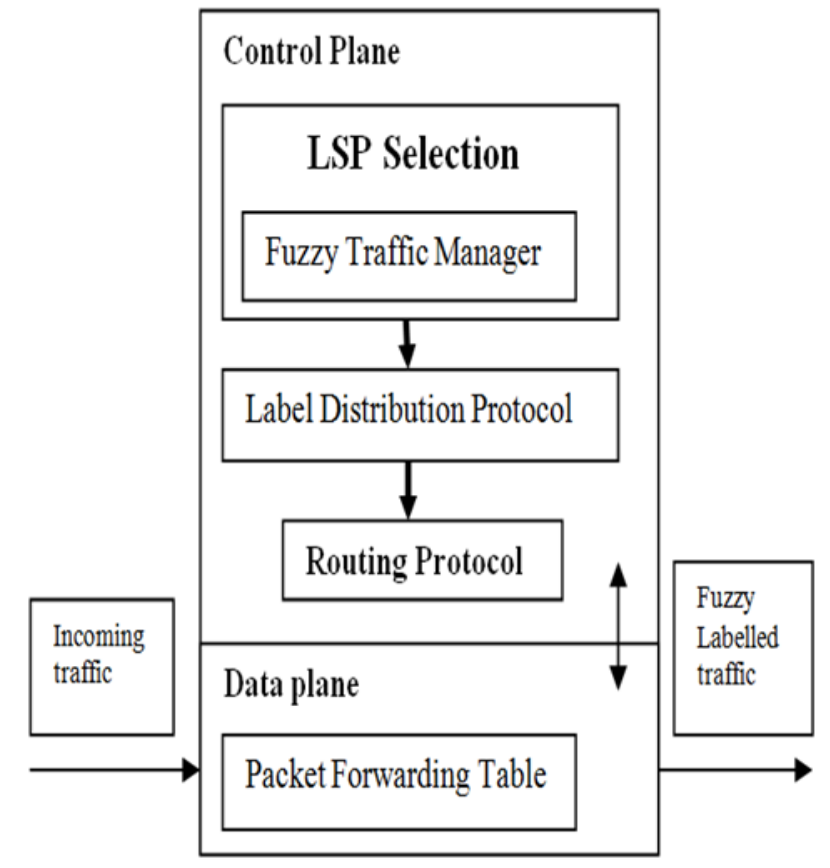

Fig. 2. The Fuzzy Traffic Manager (FTM) in the LSP selection component of an ingress node as FLCS

\begin{tabular}{|c|c|c|c|c|c|}
\hline load & VL & L & M & H & VH \\
\hline VL & Z & T & VS & S & B \\
& R2 & R3 & R4 & R5 \\
\hline L & T & VS & S & B & B \\
& R6 & R7 & R8 & R9 & R10 \\
\hline M & T & VS & S & B & B \\
& R11 & R12 & R13 & R14 & R15 \\
\hline H & VS & S & B & VB & VB \\
& R16 & R17 & R18 & R19 & R20 \\
\hline VH & S & B & B & VB & H \\
& R21 & R23 & R24 & R25 \\
\hline
\end{tabular}

Fig. 3. Fuzzy Rule Base Matrix- LsS

It provides efficient alternate paths in preference order that support Traffic Engineering and Path Optimization. The combination of rules is shown in TABLE I. The computed output response LsS_value identifies the paths.

It demonstrates the classification of LSPs and also defines the fuzzy set boundaries. Paths lying in region Z, T, VS, S, B, $\mathrm{VB}, \mathrm{H}$ are best, very good, good, satisfactory, just acceptable, not acceptable and rejected respectively. Hence, in this way it arranges the paths in preferential order for the successful transmission of packets. 
TABLE I. COMPOSITION OF RULES

\begin{tabular}{|l|l|}
\hline & Output linguistic Variables \\
\hline & Z=R1 \\
& T=R2+R6+R11 \\
& S=R3+R7+R12+R16 \\
LsS & VS=R4+R8+R13+R17+R21 \\
& B=R5+R9+R10+R14+R15+R18+R22+R23 \\
& VB=R19+R20+R24 \\
& H=R25 \\
\hline \multirow{5}{*}{ TSS } & NC=R1+R2+R3+R4+R8+R9+R10+R15+R16+R22 \\
& IO=R5+R6+R11+R12+R17+R18+R23 \\
& IW=R7+R13+R19+R24+R25+R29+R30+R20 \\
& IT=R14+R21+R26+R27+R31+R32 \\
& A=R28+R33+R34+R35 \\
\hline
\end{tabular}

TSS rule base is a set of inputs (utilization rate (UR), link capacity (LC)), with their seven linguistic values of UR (Zero (Z), Tiny (T), Very Small (VS), Small (S), Big (B), Very Big (VB), High (H)) and five linguistic values of LC (Very Low (VL), Low (L) Medium (M), High (H), Very High (VH)) and output TSS_value with their five linguistic values (Very Low (VL), Low (L) Medium (M), High (H), Very High (VH)) forms at most $7 * 5=35$ possible combinations. Hence, optimized decisions are made using developed rules 35 (R1, R2, R3... R35) as shown in Fig. 4.

For the simplicity and low computational complexity of operation, Triangular Membership Function (TMF) is applied to obtain the membership function of metrics as:-

triangle $(x ; a, b, c)=\max (\min (x-a / b-a, c-x / c-b), 0)$,

It is defined by three parameters, where $b$ indicates the point on which, the membership function value is 1 , a and c indicate the left and right limits of the definition domain of the membership function.

On applying TMF as shown in formula (1), we obtain membership functions of input and output parameters as follows:-

$\mu_{\text {load }}=\left\{\mu_{\mathrm{VL}}, \mu_{\mathrm{L}}, \mu_{\mathrm{M}}, \mu_{\mathrm{H}}, \mu_{\mathrm{VH}}\right\}$,

$\mu_{\text {delay }}=\left\{\mu_{\mathrm{VL}}, \mu_{\mathrm{L}}, \mu_{\mathrm{M}}, \mu_{\mathrm{H}}, \mu_{\mathrm{VH}}\right\}$,

$\mu_{\text {LsS_value }}=\left\{\mu_{\mathrm{Z}}, \mu_{\mathrm{T}}, \mu_{\mathrm{Vs}}, \mu_{\mathrm{S}}, \mu_{\mathrm{B},}, \mu_{\mathrm{VB}}, \mu_{\mathrm{H}}\right\}$,

$\mu_{\text {utilization_orate }}=\left\{\mu_{\mathrm{Z}}, \mu_{\mathrm{T}}, \mu_{\mathrm{Vs}}, \mu_{\mathrm{s}}, \mu_{\mathrm{B},} \mu_{\mathrm{VB},} \mu_{\mathrm{H}}\right\}$,

$\mu_{\text {link_capacity }}=\left\{\mu_{\mathrm{VL}}, \mu_{\mathrm{L}}, \mu_{\mathrm{M}}, \mu_{\mathrm{H}}, \mu_{\mathrm{VH}}\right\}$,

$\mu_{\text {TSS_value }}=\left\{\mu_{\mathrm{NC}}, \mu_{\mathrm{IO}}, \mu_{\mathrm{IW}}, \mu_{\mathrm{IT}}, \mu_{\mathrm{A}}\right\}$.

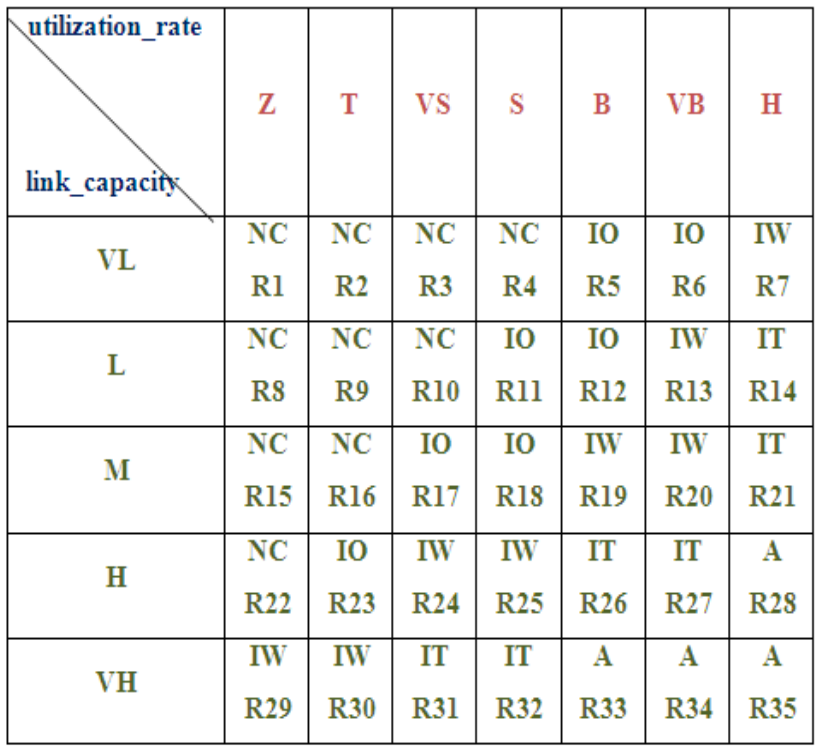

Fig. 4. Fuzzy Rule Base Matrix- TSS

TABLE II. DEFUZZIFICATION OF FUZZY CONTROLLERS

\begin{tabular}{|c|c|c|c|}
\hline & $\begin{array}{l}\text { Linguistic } \\
\text { term }\end{array}$ & $\begin{array}{l}\text { Linguistic } \\
\text { center }\end{array}$ & \\
\hline LsS & $\begin{array}{c}\text { Z } \\
\text { T } \\
\text { VS } \\
\text { S } \\
\text { B } \\
\text { VB } \\
\text { H }\end{array}$ & $\begin{array}{c}\mathbf{Z}_{\mathrm{C}} \\
\mathbf{T}_{\mathrm{C}} \\
\mathrm{VS}_{\mathrm{C}} \\
\mathrm{S}_{\mathrm{C}} \\
\mathbf{B c} \\
\mathbf{V B C} \\
\mathbf{H}_{\mathrm{C}}\end{array}$ & $\begin{array}{c}\text { LsS_value }=\left(\mathrm{Z}^{*} \mathbf{Z}_{\mathrm{C}}\right. \\
+\mathrm{T}^{*} \mathbf{T}_{\mathrm{C}}+\mathrm{VS}^{*} \mathrm{VS}_{\mathrm{C}} \\
+\mathrm{S}^{*} \mathrm{~S}_{\mathrm{C}}+\mathbf{B}^{*} \mathbf{B}_{\mathrm{C}} \\
\left.+\mathrm{VB}^{*} \mathrm{VB}_{\mathrm{C}}+\mathbf{H}^{*} \mathbf{H}_{\mathrm{C}}\right) / \\
(\mathrm{Z}+\mathrm{T}+\mathrm{VS}+\mathrm{S}+\mathbf{B}+\mathrm{VB} \\
+\mathbf{H})\end{array}$ \\
\hline TsS & $\begin{array}{l}\text { NC } \\
\text { IO } \\
\text { IW } \\
\text { IT } \\
\text { A }\end{array}$ & $\begin{array}{c}\mathbf{N C}_{\mathbf{C}} \\
\mathbf{I O}_{\mathrm{C}} \\
\mathbf{I W}_{\mathrm{C}} \\
\mathbf{I T}_{\mathrm{C}} \\
\mathbf{A}_{\mathrm{C}}\end{array}$ & $\begin{array}{c}\text { TSS_value }=\left(\mathbf{N C}^{*}\right. \\
\mathrm{NC}_{\mathrm{C}}+\mathbf{I O}^{*} \mathbf{I O}_{\mathrm{C}} \\
+\mathbf{I W}^{*} \mathbf{I W}_{\mathrm{C}}+\mathbf{I T}^{*} \\
\left.\mathbf{I T}_{\mathrm{C}}+\mathbf{A}^{*} \mathbf{A}_{\mathrm{C}}\right) / \\
(\mathbf{N C}+\mathbf{I O}+\mathbf{I W}+\mathbf{I T}+\mathbf{A})\end{array}$ \\
\hline
\end{tabular}


3) Pseudo code of FLCS

The algorithm 1 depicts packet forwarding process. As, FLCS works according to two fuzzy controllers Label setup System (LsS) and Traffic Splitting System (TSS).

An Algorithm 1 is basically the main program which calls the LsS and TSS as shown in TABLE III. It conclusively responsible for the packet forwarding by maintaining the list of LSPs arranged in preference order obtained from LsS and also performs traffic splitting among the links obtained as Lused.

Algorithm 2 and 3 reveals the executions of these controllers respectively.

Algorithm 2 reads the updated value of the load (L) and calculates delay (D) for all links/ LSPs as shown in TABLE VI. After applying complete the procedure of fuzzy logic, LsS_value of all total LSPs (TLSPS) is obtained. After sorting these values, LSPs are arranged in preference order of selection for routing as SLSPs.

TABLE III. Algorithm 1 Executes Packet ForWARding Process IN INGRESS NODE

Input: IPacket, incoming traffic packet arriving at the

$$
\text { ingress node }
$$

Precondition: Traffic Constraints values are to be fed in

FTM,LDP is session control protocol

\section{Main program :}

1. If request packet arrives at ingress

2. Call LsS and TSS

3. If request packet $=$ NULL

then terminate

else

goto step $2 \& 3$

\section{End if}

TABLE IV. ALGORITHM 2 EXECUTES LSS IN FLCS

Input: QoS Constraints values are to be fed in FTM

Precondition: Updated link constraints of $\mathrm{L}[\mathrm{i}][\mathrm{j}], \mathrm{D}[\mathrm{i}] \mathrm{j}]$ and

$\mathrm{LC}[\mathrm{i}][\mathrm{j}]$

Main program :

1. For $\mathrm{i}=1$ to $\mathrm{n}$ do

2. For $\mathrm{j}=1$ to $n$ do

3. $\quad$ Read L[i][j]

4. EndFor

5. EndFor

6. For $\mathrm{i}=1$ to $n$ do

7. $\quad$ For $\mathrm{j}=1$ to $n$ do

8. $\mathrm{D}[\mathrm{i}][\mathrm{j}]=\mathrm{L}[\mathrm{i}][\mathrm{j}] / \mathrm{LC}[\mathrm{i}][\mathrm{j}]$

9. EndFor

10. EndFor

11. LsS_value $(i, j)=L s S(L, D)$

12. For $m=1$ to TLSPs

13. SLSPs $[\mathrm{m}]=\operatorname{sort}\left(\min \left(\operatorname{LsS} \_v a l u e(i, j)\right)\right)$

14. Return SLSPs[m]

\section{EndFor}

An algorithm 3 reads the updated value of link capacity (LC) and evaluates an utilization rate (UR) of each LSP dynamically as shown in TABLE V. The frequency (Freq) of utilized LSP is recorded as Lused. After applying the complete procedure of fuzzy logic, TSS_value is obtained. This resultant fuzzy value tells how many LSPs are required for congestion free network as NLSPs. 
TABLE V. ALGORITHM 3 EXECUTES TSS IN FLCS

Input: Traffic Constraints values are to be fed in FLCS

Precondition: $L C[\mathrm{i}][\mathrm{j}]$ of links from LIB

\section{Main program :}

1. For $\mathrm{i}=1$ to $\mathrm{n}$ do

2. For $\mathrm{j}=1$ to $\mathrm{n}$ do

3. $\quad \operatorname{Read} L C[i][j]$

4. EndFor

5. EndFor

6. For $\mathrm{i}=1$ to $\mathrm{n}$ do

7. For $\mathrm{j}=1$ to $\mathrm{n}$ do

8. Freq $[\mathrm{i}][\mathrm{j}]=\operatorname{Lused}[\mathrm{i}][\mathrm{j}]$

9. Freq $[\mathrm{i}][\mathrm{j}]=$ Freq $[\mathrm{i}][\mathrm{j}]+1$

10. UR $[\mathrm{i}][\mathrm{i}]=$ Freq $[\mathrm{i}][\mathrm{j}] / \operatorname{Lused}[\mathrm{i}][\mathrm{j}]$

11. EndFor

12. EndFor

13. TSS_value $(\mathrm{i}, \mathrm{j})=\mathrm{TSS}(\mathrm{UR}, \mathrm{LC})$

14. Return NLSPs

15. EndFor

\section{SIMULATION ANALYSIS AND RESULTS}

For validating incremental progress related to solving the problem of traffic splitting, a comparison between classical approach (OSPF) and proposed work (FLCS) was conducted by multiple experiments using NS-3[18-26]. We have performed a group of simulations in the scenario composed of MPLS-TE together with constraint based routing. FTM performs the dynamic route selection. Taking this as scenario, our work demonstrates the improvements by adding FTM for LSP Selection. The topology used to evaluate the proposed work behavior consists of three different link capacities and propagation delay as follows in Fig 5:- a) $10 \mathrm{Mbps}$, 3ms (Red) b) 100 Mbps, 2 ms (Green) c) 1000 Mbps, 1ms(Black).
The incoming requests arriving follow an exponential distribution ( $\lambda$ ) and the requested bandwidth is uniformly distributed between ranges [0 10] (Mbps), [10 100] (Mbps), [100 1000] (Mbps) to model Voice, Data and Video Traffic respectively $[27,28]$. The holding time is randomly distributed with a mean of $300 \mathrm{sec}(\mathrm{ON}=0.325 \mathrm{OFF}=.64)$.

IPV4 network stack has been used and packets are generated using a hypothetical ON/OFF model using the Drop tail queue. Different traffic types are used in simulations like the FTP over the TCP to simulate data traffic and the constant bit rate (CBR) to simulate voice and video over the UDP. The packet size of the User Datagram Protocol (UDP) is 512 byte and 1024 byte for the Transport Control Protocol (TCP). Finally, the existing topology and an available resources database in ingress assumed perfectly updated. The statistical comparison of FLCS and OSPF is depicted in TABLE VI, which illustrates that standard deviation for different traffic is high in the case of OSPF is obtained with varying UDP traffic for 300 seconds.

In the Fig. 6 (a,b) the mean delay and the mean loss rate of video traffic is displayed, which is obtained with varying UDP traffic for 300 seconds. In the Fig. 6 (c,d) the mean delay and mean loss rate of data traffic is displayed, which is obtained with varying UDP traffic for 300 seconds. In the Fig. 6 (e, f) the mean delay and the mean loss rate of Voice traffic is displayed, which is obtained with varying UDP traffic for 300 seconds. Moreover, $42 \%, 15.4 \%$ and $44.4 \%$ of Video packets, Data packets and Voice packets respectively are dropped due to buffer overflow at the congested node when using OSPF. FLCS on the other hand, as seen from Fig. 6 by avoiding this congestion as mentioned earlier, maintains the traffic delivery performance.

By distributing the traffic over suitable preferable paths and maintaining low queue lengths, all the packets can be successfully delivered. The result is an increase in the queuing delay at a particular congested node, consequently leading congestion, and large waiting time at a few hops where the performance is poor. For few seconds in the above scenarios, OSPF outperforms due to low queuing delay. Even though FLCS utilizes multiple paths and it leads to less delay for the said Congested path. The obtained results of simulation experiments are displayed in as Fig. 6 and Table VI gives the better understanding of research work. 


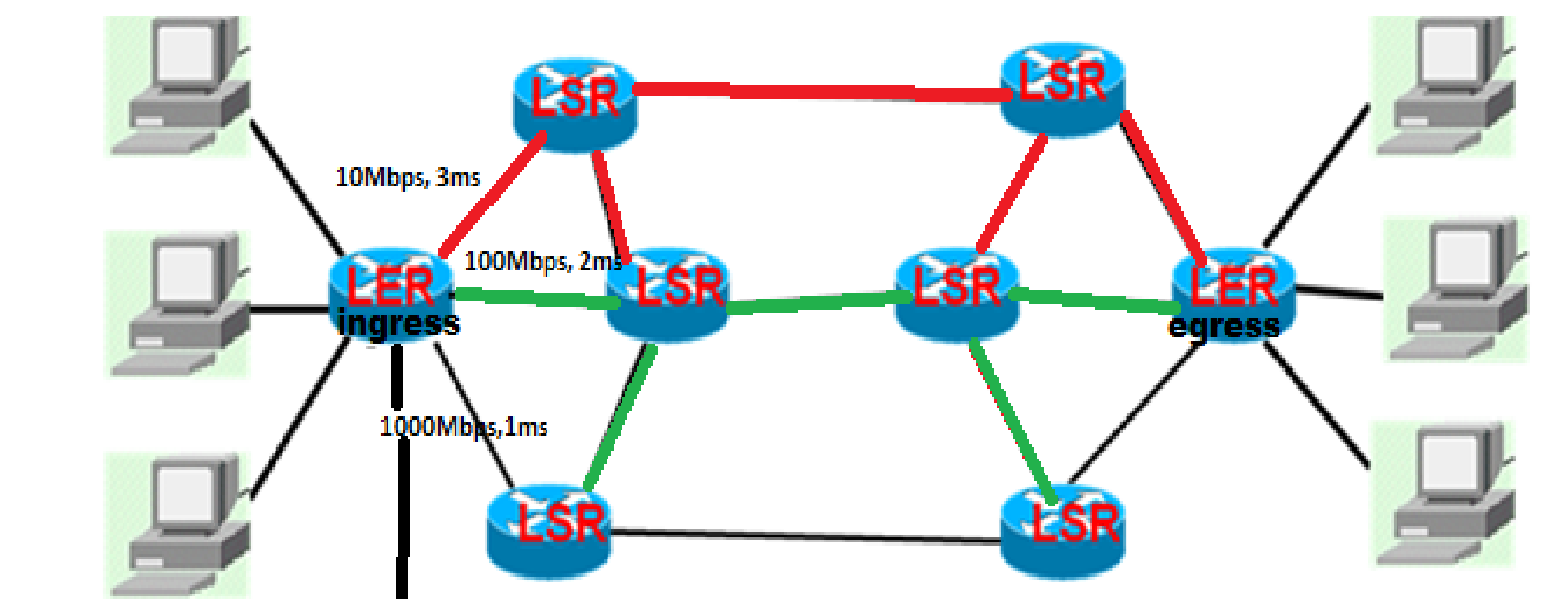

Traffic sources

Destination

INGRESS

Fig. 5. Link capacities and propagation delays a) 10 Mbps, 3ms (Red) b)100 Mbps, 2 ms(Green) c)1000 Mbps, 1ms(Black)

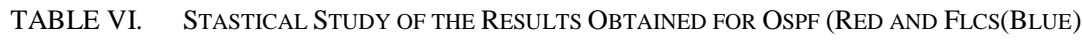

\begin{tabular}{|c|c|c|c|c|c|c|c|c|}
\hline \multicolumn{3}{|c|}{ Video Traffic } & \multicolumn{3}{|c|}{ Data Traffic } & \multicolumn{3}{|c|}{ Voice Traffic } \\
\hline MAX & 33.6 & 29.4 & MAX & 0.16 & 8.604 & MAX & 19.1 & 14.9 \\
\hline MIN & 0 & 0 & MIN & 0.0011 & 0.001 & MIN & 0 & 0 \\
\hline \multicolumn{3}{|c|}{ Mean Delay } & \multicolumn{3}{|c|}{ Mean Delay } & \multicolumn{3}{|c|}{ Mean Delay } \\
\hline MAX & 33.6 & 29.4 & MAX & 0 & 0.126 & MAX & 0.25 & 0.21 \\
\hline MIN & 0 & 0 & MIN & 0.0011 & 0.001 & MIN & 0 & 0 \\
\hline
\end{tabular}

VI. CONCLUSIONS

Our premise of this research is based on the fuzzy traffic manager which works within the realims of MPLS. The FTM is positioned at ingress node. The proposed fuzzy based approach is highly suitable for congestion control over MPLS networks. It applies QoS \& TE constraints. It is designed for better video delivery, since it provides better throughput, average delay. We also empirically demonstrated the advantages of FLCS over OSPF in NS-3.

In current simulation, the network was using 2 edge routers and 6 switch routers. We achieved significant improvement as $42 \%, 15.4 \%$ and $44.4 \%$ of Video packets, Data packets and Voice packets respectively are dropped due to buffer overflow at the congested node when using OSPF. It is applicable for any kind of traffic, where traffic volume is small and traffic type is normal. However, it is based on traffic scenarios, which have been implemented in this research paper. This work gives an opportunity to analyze the complex scenario in network traffic management, which would help future implementation in this context. As multipath routing is the case of uneven length of paths. The current approach demonstrated here, was able to track load, delay as well as link capacity for taking decisions.
So the framework is capable of performing non-redundant search and tracking in order to perform congestion free traffic flow. It is an artificial intelligence based self-configurable framework with enhanced scalability. No such framework exists in literature that can simultaneously perform routing and traffic splitting using fuzzy predictions. It was found that fuzzy approach used here, is performing better as compared to previous work. In this way, we were able to build a "soft controller" supporting a "hardware controller", as traffic splitting, traffic flow control is better, more responsive in nature. These findings predict that the fuzzy traffic monitor proved as more efficient solution and constitute a good alternative to some conventional methods of traffic engineering based on complex optimization.

\section{FUTURE DiRECTIONS}

In Future, work can be carried out to validate the approach in more simulated dynamic scenarios. The interest of such a work has a big impact for the applications related to the networks, in particular those dedicated to the mission critical application. 


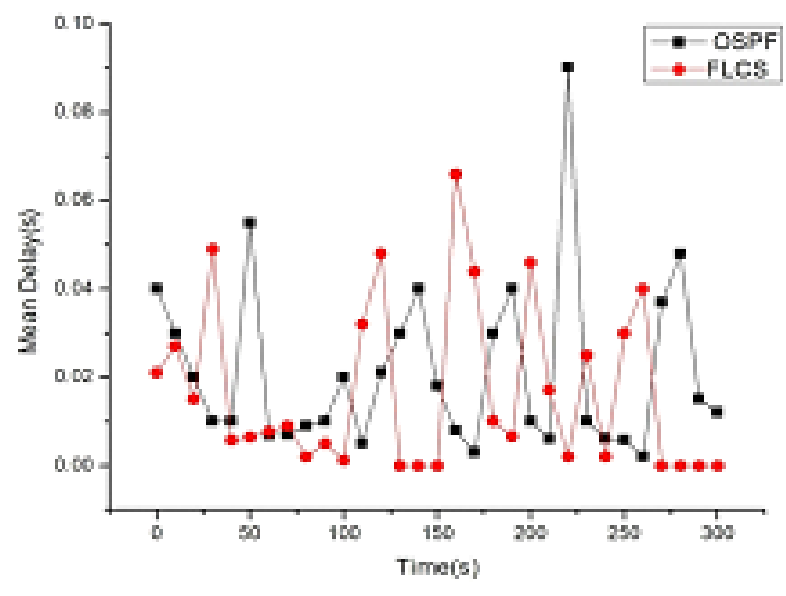

(a)

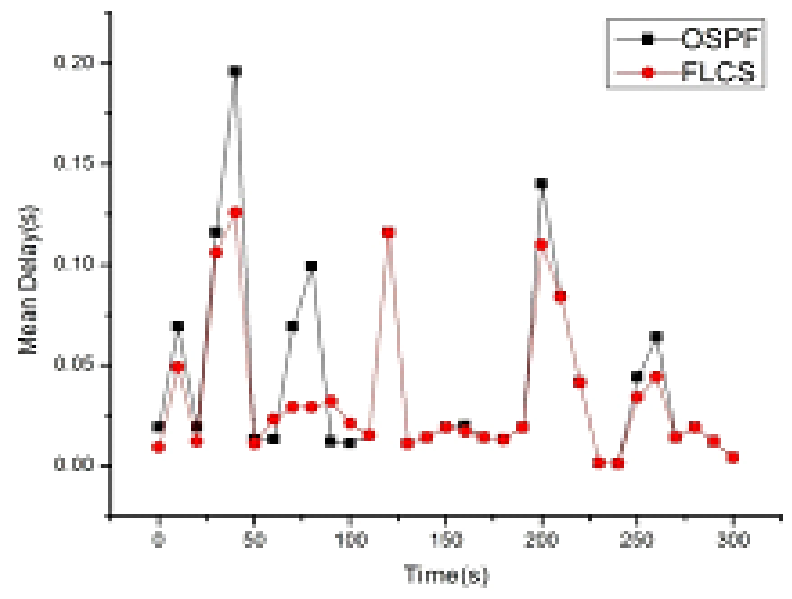

[c]

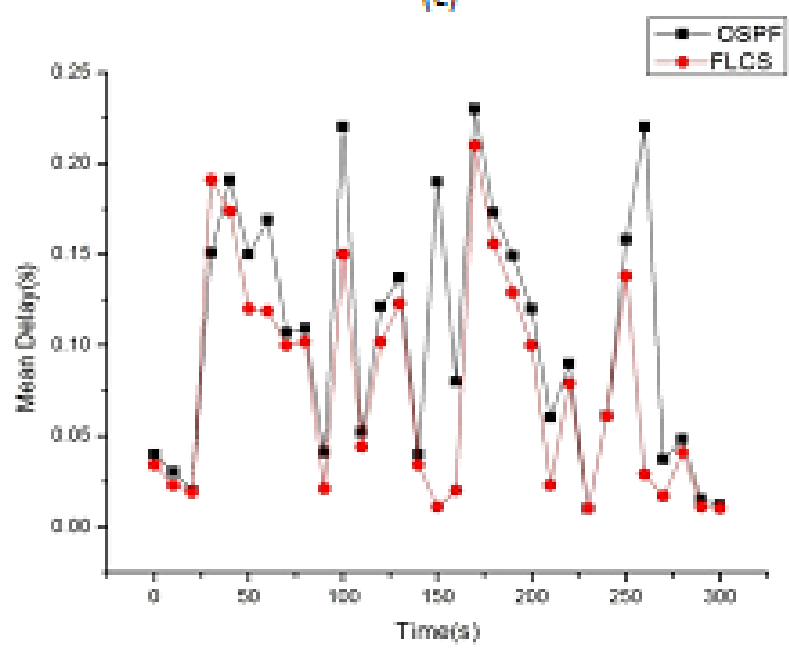

[e]

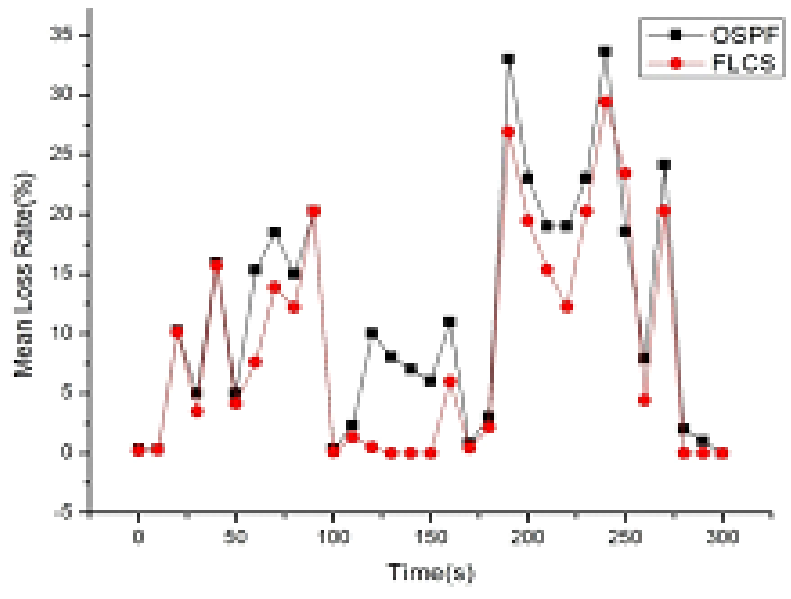

(b)

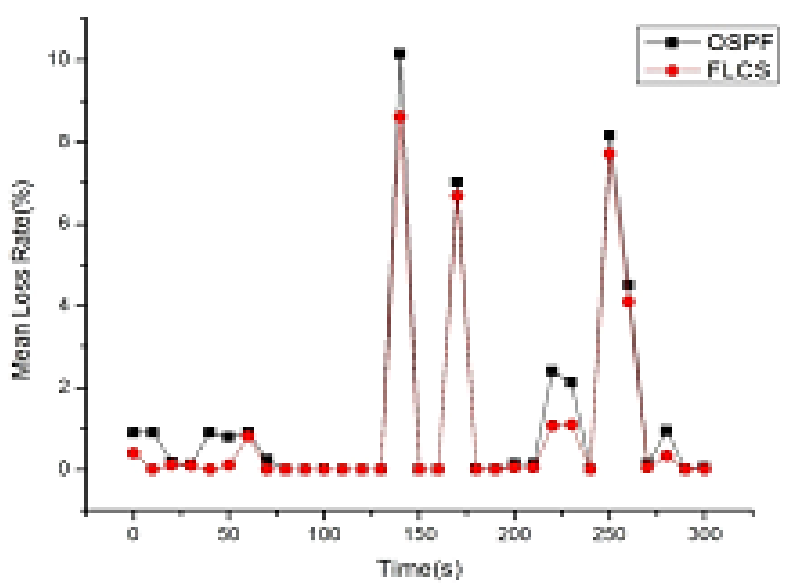

(d)

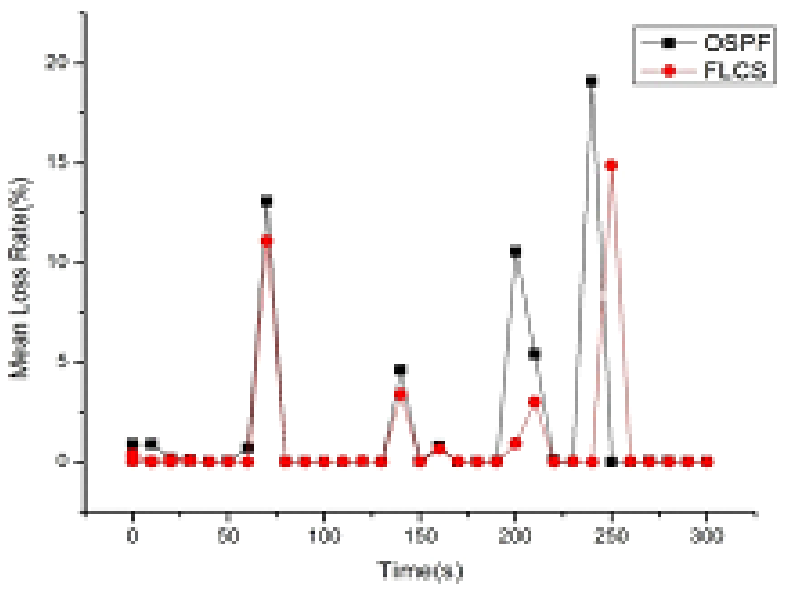

(f)

Fig. 6. (a)(b) Variation of Mean delay(s) and Mean Loss Rate (\%) for Video Traffic with respect to time(secs) (c)(d) Variation of Mean delay(s) and Mean Loss Rate (\%) for Data Traffic with respect to time (secs)

(e)(f) Variation of Mean delay(s) and Mean Loss Rate (\%) for Voice Traffic with respect to time (secs) 


\section{REFERENCES}

[1] Awduche, D. O., “Applicability statement for extensions to RSVP for LSP-tunnels”, 2001.

[2] Chung, Jong-Moon, Sang-Chul Kim, Elie Marroun, and Harman Sandhu. "VoIP over MPLS networking requirements." In Networking-ICN 2001, Springer Berlin Heidelberg, pp. 735-744, 2001.

[3] Verchere, D., \& Berde, B.. The Advances in Control and Management for Transport Networks. Core and Metro Networks, pp.55-126, 2010.

[4] Rahim-Amoud, R., Merghem-Boulahia, L., \& Gaiti, D., "Towards an Adaptive and Intelligent MPLS Network in Network Control and Engineering for QoS”, Security and Mobility, IV, Springer US, pp. 209214, 2007.

[5] Sharma, Nonita, and Ajay K. Sharma. "Comparative analysis of SIR and SID in Wireless Sensor Networks using temporal correlation." In Computing, Communication \& Automation (ICCCA), 2015 International Conference on, pp. 464-468. IEEE, 2015.

[6] Sharma, Nonita, Ajay K. Sharma, and Kumar Shashvat. "Impact of Heterogeneous Deployment on Source Initiated Reactive Approach."International Journal of Advanced Computer Science \& Applications 1, no. 6: 185-191.

[7] Katz, D., Kompella, K., \& Yeung, D., “Traffic engineering (TE) extensions to OSPF version 2”, RFC 3630, September, 2003.

[8] Pan, P., Swallow, G., \& Atlas, A., “ Fast reroute extensions to RSVP-TE for LSP tunnels”, RFC 4090, 2005.

[9] Halabi, W., Steenhaut, K., Goossens, M., Truong, T. H., \& Nowé, A., "Hierarchical routing and traffic grooming in IP/MPLS-based ASON/GMPLS multi-domain networks “. Photonic Network Communications, 23(3), 217-229, 2012.

[10] Majd, N. E., \& Yaghmaee, M. H., “A fuzzy algorithm for QoS-based routing in MPLS network”, In Communications, 2006. APCC'06. AsiaPacific Conference,. IEEE, pp. 1-5 August, 2006.

[11] Zhou, B., \& Mouftah, H. T. , “Adaptive shortest path routing in GMPLS-based optical networks using fuzzy link costs”, In Electrical and Computer Engineering, 2004. Canadian Conference, IEEE,Vol. 3, pp. 1653-1657, May, 2004.

[12] Hsu, W. H., Shieh, Y. P., \& Chen, J., "Multiple path selection algorithm for DiffServ-aware MPLS traffic engineering” Computer Communications,33(13), 1557-1565, 2010.

[13] Kirov, G., \& Lakov, D., “Soft Computing Agents for MPLS Networks”, Cybernetics and Information Technologies, 2(2), 2002.

[14] Kar, K., Kodialam, M., \& Lakshman, T. V., "Minimum interference routing of bandwidth guaranteed tunnels with MPLS traffic engineering applications" .Selected Areas in Communications, IEEE Journal on, 18(12), 2566-2579, 2000.

[15] Mérindol, P., François, P., Bonaventure, O., Cateloin, S., \& Pansiot, J. J., "An efficient algorithm to enable path diversity in link state routing networks”. Computer Networks, 55(5), 1132-1149, 2011.

[16] Aminian, M., Alavi, S. E., \& Tahan, M. N.,” Differentating services using cross-layer fuzzy-based queuing in IP networks", In Telecommunications (IST), 2014 7th International Symposium on IEEE, pp. 652-657, September, 2014.
[17] Zadeh, L.A., "Fuzzy sets", Information and Control 8 (3): PP. 338-353, 1965.

[18] Choi, B. Y., Zhang, Z. L., \& Du, D. H. C., "Scalable Network Monitoring in High Speed Networks", Springer Science \& Business Media, 2011.

[19] Kurose and Ross, "Computer Networks Delay, Loss and Throughput Delay, Loss and Throughput Layered architectures Layered architectures Based on Computer Networking”. Pearson/Addison Wesley, 2008.

[20] Núñez-Martínez, J., Baranda, J., \& Mangues-Bafalluy, J. , "Experimental evaluation of self-organized backpressure routing in a wireless m esh backhaul of small cells”, Ad Hoc Networks, 24, 103-114, 2015.

[21] Sydney, A., Nutaro, J., Scoglio, C., Gruenbacher, D., \& Schulz, N., "Simulative comparison of multiprotocol label switching and openflow network technologies for transmission operations Smart Grid”, IEEE Transactions on,4(2), pp. 763-770, 2013.

[22] Sydney, A., Ochs, D. S., Scoglio, C., Gruenbacher, D., \& Miller, R. Using GENI for experimental evaluation of Software Defined Networking in smart grids. Computer Networks, 63, pp. 5-16, 2014.

[23] Carneiro, G., Fortuna, P., \& Ricardo, M., FlowMonitor: a network monitoring framework for the network simulator 3 (NS-3). In Proceedings of the Fourth International ICST Conference on Performance Evaluation Methodologies and Tools (p. 1). ICST (Institute for Computer Sciences, Social-Informatics and Telecommunications Engineering), October, 2009.

[24] Carneiro, G., Fontes, H., \& Ricardo, M. "Fast prototyping of network protocols through ns-3 simulation model reuse”. Simulation Modelling Practice and Theory, 19(9), pp. 2063-2075, 2011.

[25] Da Fonseca, S., "Bandwidth allocation in self-sizing networks under uncertain constraints". In 2006 IEEE International Conference on Communications, Vol. 2, pp. 681-686, 2006.

[26] Drummond, A. C., da Fonseca, N. L., \& Devetsikiotis M., “A multiobjective fuzzy bandwidth partitioning model for self-sizing networks”. European Journal of Operational Research, 191(3), pp. 11611174, 2008.

[27] Sánchez-López, S., Masip-Bruin, X., Solé-Pareta, J., \& Domingo Pascual J., "Providing QoS in MPLS-ATM integrated environment in from QoS Provisioning to QoS Charging”, Springer Berlin Heidelberg , pp. 215-224, 2002.

[28] Genge, B., \& Siaterlis, C., "Analysis of the effects of distributed denialof-service attacks on MPLS networks", International Journal of Critical Infrastructure Protection, 6(2), pp. 87-95, 2013.

[29] Adas, A., "Traffic models in broadband networks". Communications Magazine, IEEE, 35(7), pp. 82-89, 1997.

[30] Park, K., \& Willinger, W., "Self-similar network traffic: An overview. Self-similar network traffic and performance evaluation”, pp. 1-38, 2000.

[31] Liu, H. A., Pradeep, V., \& Kini, S., U.S. Patent No. 8,339,942. Washington, DC: U.S. Patent and Trademark Office, 2012. 